\title{
DC IR-Drop Analysis of Multilayered Power Distribution Network by Discontinuous Galerkin Method with Thermal Effects Incorporated
}

\author{
Ping Li, Senior Member, IEEE, Min Tang, Member, IEEE, Zhi Xiang Huang, Member, IEEE, Li \\ Jun Jiang, Fellow, IEEE, and Hakan Bağcı, Senior Member, IEEE
}

\begin{abstract}
Due to the temperature dependent resistivity of power delivery network (PDN) interconnects, a wiser and necessary strategy is to proceed the electrical-thermal co-simulation in order to include the thermal effects caused by Joule Heating. As a natural domain decomposition method (DDM), in this work, a discontinuous Galerkin (DG) method is proposed to facilitate the steady-state electrical and thermal co-analysis. With the intention to avoid solving a globally coupled steady-state matrix system equations resulted by the implicit numerical flux in DG, the block Thomas method is deployed to solve the entire domain in a subdomain by subdomain scheme. As a direct solver, the block Thomas method is free of convergence problem frequently occurring in iterative methods such as block GaussSeidel method. The capability of the proposed DG method in handling multiscale and complex 3D PDNs is validated by several representative examples.
\end{abstract}

Index Terms-Discontinuous Galerkin (DG) method, DC IRdrop, electrical-thermal co-simulation, integrated circuits (IC), power delivery network (PDN), steady-state.

\section{INTRODUCTION}

$\mathbf{T}$ HE continuing decrease in DC supplied voltage to the chips and switching circuits requires more efforts in managing the ripple across the power distribution network (PDN) [1], while the ever rising integration capacity increases the current density, which brought significant challenges to the design of PDNs in order to achieve the intended power integrity (PI) as well as the signal integrity (SI) performance. Moreover, the nonideal resistance of PDN interconnects further deteriorates the PI performance due to the Joule Heating, where the thermal effects not only increase the DC voltage drop, but also possibly put the high-speed signals under

This work is supported by National Natural Science Foundation of China (NSFC) under Grant 61701423, 61674105, 61831016, 61722101, 61701424 , and in part by National Key Research and Development Program of China under Grant 2019YFB1802904 and 2019YFB2205001. (Corresponding author: Ping Li and Min Tang)

P. Li and M. Tang are with the Key Laboratory of Ministry of Education of China for Research of Design and Electromagnetic Compatibility of High Speed Electronic Systems, Shanghai Jiao Tong University, Shanghai, China. Ping Li is also with HKU-SIRI, Shenzhen, China. (ping.li@sjtu.edu.cn,tm222@sjtu.edu.cn)

Z. X. Huang is with the Institute of Physical Science and Information Technology, Anhui University.

L. J. Jiang is with the Department of Electrical and Electronic Engineering, The University of Hong Kong, Hong Kong SAR., China.

H. Bağc1 is with the Division of Computer, Electrical, and Mathematical Sciences and Engineering, King Abdullah University of Science and Technology (KAUST), Thuwal, 23955-6900, Saudi Arabia. risk. Thereby, it is excessively necessary and important to implement the electrical-thermal co-simulation [2] in order to maintain the performance of the whole integrated circuits (IC). With this objective in mind, various approaches have been proposed in the past few years.

The first kind of approaches for the DC IR-drop analysis are on the basis of equivalent circuit models [3], [4] in terms of finite difference discretization, in which the discretized Laplace equation governing the potential is firstly transformed into a $2 \mathrm{D}$ one and then it is further approximated by a resistive network. However, the IR-drop is lack of consideration of the thermal effect.

To capture the thermal effects, soon after, numerical algorithms such as finite volume method (FVM) [5] and finite element method (FEM) [6]-[8] are proposed to solve the electrical and the thermal equations together. Due to the temperature dependent material properties, the two equations are solved in an iterative scheme.

In this work, a discontinuous Galerkin (DG) method [9][11] is proposed to conduct the rigorous DC-IR drop analysis with thermal effects included. As the combination of FVM and FEM, DG method inherits the advantages of FVM and FEM. Like FVM [12], solutions in the adjacent subdomains communicate with each other by the numerical flux, it is thus a natural domain decomposition method (DDM); on the other hand, it is capable of flexibly making use of high-order basis functions to approximate the solution, thereby like FEM [13], it is able to achieve high-order accuracy. However, for the DC-IR drop analysis, both the electrical and the thermal equations are in a steady-state, the numerical flux used for information exchange is in an implicit form, consequently resulting in a globally coupled matrix equation, which dramatically complicates the problem. To beat this deficiency, the whole computational domain is firstly tessellated into a number of non-overlapping subdomains in a nested sequence, and then the system matrix equation can be formulated into a block tridiagonal matrix system where the upper and the lower blocks resulted from the incoming numerical flux represent couplings between the neighboring subdomains, and finally the block Thomas method [14] is applied to solve the implicitly coupled matrix system in a subdomain by subdomain scheme. Thereby, the advantage possessing by the explicit DG algorithm is kept, namely, it only needs to solve a number of smaller matrix equations instead of a global one. 


\section{ElECTRICAL-THERMAL CO-ANALYSiS WITH DG FORMULATION}

\section{A. Electrical Equation}

Based on the vector form of Ohm's law, for the DC-IR drop analysis, the current density $\mathbf{J}$ in a conductor is defined as

$$
\mathbf{J}=\sigma \mathbf{E}
$$

where $\sigma$ is the electrical conductivity of the conductor, and the electric field $\mathbf{E}$ can be expressed as a function of electrical potential $\phi$, namely,

$$
\mathbf{E}=-\nabla \phi
$$

With (2), (1) can be reformulated into

$$
\mathbf{J}=-\sigma \nabla \phi
$$

Further resorting to the equation of continuity of electric charge, the current density $\mathbf{J}$ should satisfies the divergence free condition given by

$$
\nabla \cdot \mathbf{J}=0
$$

Based on the governing equations in (3) and (4), the current density as well as the potential distribution over the whole PDN can be uniquely determined in terms of the following boundary conditions:

$$
\begin{aligned}
& \phi=\phi_{0} \quad \text { on } \quad \Gamma_{\mathrm{dc}} \\
& \hat{\mathbf{n}} \cdot \mathbf{J}=\frac{\phi}{R_{L} S} \quad \text { on } \Gamma_{R}
\end{aligned}
$$

where $\Gamma_{\mathrm{dc}}$ is the Dirichlet boundary surface with voltage already given, $\Gamma_{R}$ is the impedance boundary surface connecting with external load such as decoupling capacitors, match network, chips and memories, $R_{L}$ is the resistance value of the external equivalent load, and $S$ is the area of the cross section of the impedance boundary surface.

In order to solve (4) and (3) by the proposed DG method, the computational domain $\Omega$ is firstly decomposed into a number of non-overlapping and conformal subdomains $\Omega_{k}$ $(k=1, \cdots, K)$, and each subdomain is further teared into a series of tetrahedrons $\Omega_{k}^{i}\left(i=1, \cdots, K_{k}\right)$, then the Galerkin test is applied to (4) and (3) pertinent to the $k$-th subdomain, which leads to the following two new formulations:

$$
\begin{aligned}
& \int_{\Omega_{k}} \nabla \varphi_{p}^{k} \cdot \mathbf{J}^{k} d V=\int_{\Gamma_{k l}} \varphi_{p}^{k}\left(\hat{\mathbf{n}} \cdot \tilde{\mathbf{J}}^{k}\right) d S \\
& \int_{\Omega_{k}} \psi_{\xi, q}^{k} \cdot J_{\xi}^{k} d V=\int_{\Omega_{k}} \sigma^{k} \frac{\partial \psi_{q}^{k}}{\partial_{\xi}} \cdot \phi^{n} d V-n_{\xi} \int_{\Gamma_{k l}} \sigma^{k} \psi_{\xi, q}^{k} \cdot \tilde{\phi}^{k} d S
\end{aligned}
$$

where $\varphi_{p}^{k}$ and $\psi_{q}^{k}$ are the $p$-th and the $q$-th nodal basis functions for the potential and the current density, respectively; $\hat{\mathbf{n}}$ is an unit normal vector pointing from the present subdomain $k$ to its neighboring subdomain $l, \tilde{\mathbf{J}}^{k}$ and $\tilde{\phi}^{k}$ are called numerical flux defined by [15]

$$
\begin{aligned}
& \hat{\mathbf{n}} \cdot \tilde{\mathbf{J}}^{k}=C_{10}\left(\hat{\mathbf{n}} \cdot \mathbf{J}^{k}+\hat{\mathbf{n}} \cdot \mathbf{J}^{l}\right)+C_{11}\left(\hat{\mathbf{n}} \cdot \mathbf{J}^{k}-\hat{\mathbf{n}} \cdot \mathbf{J}^{l}\right) \\
& \quad+C_{12}\left(\phi^{k}-\phi^{l}\right)
\end{aligned}
$$

$$
\begin{aligned}
& \tilde{\phi}^{k}=C_{20}\left(\phi^{k}+\phi^{l}\right)+C_{21}\left(\hat{\mathbf{n}} \cdot \mathbf{J}^{k}-\hat{\mathbf{n}} \cdot \mathbf{J}^{l}\right) \\
& +C_{22}\left(\phi^{k}-\phi^{l}\right)
\end{aligned}
$$

In this work, the upwind flux is employed, where the parametric coefficients are defined as: $C_{10}=0.5, C_{11}=0$, $C_{12}=-4, C_{20}=0.5, C_{21}=0$, and $C_{22}=0$.

To obtain a fully discrete matrix system, next, the potential $\phi^{k}$ and the current density $\mathbf{J}^{k}$ are further approximated by nodal basis functions:

$$
\begin{aligned}
\phi^{k} & =\sum_{p=1}^{P^{k}} \gamma_{p}^{k} \varphi_{p}^{k} \\
J_{\xi}^{k} & =\sum_{q=1}^{Q_{\xi}^{k}} \nu_{\xi, q}^{k} \psi_{\xi, q}^{k}
\end{aligned}
$$

where $P^{k}$ and $Q_{\xi}^{k}$ denote the total number of basis functions for the expansion of potential $\phi^{k}$ and current density $\mathrm{J}_{\xi}^{k}(\xi=$ $x, y, z)$ in the $k$-the subdomain, respectively; $\gamma_{p}^{k}$ and $\nu_{\xi, q}^{k}$ are the corresponding expansion coefficients to be determined.

Substituting (7) to (10) into (5) and (6), the following matrix equations can be reached,

$$
\begin{aligned}
& \mathbf{M}_{\phi j}^{k} \boldsymbol{\nu}^{k}+\mathbf{F}_{\phi j}^{k k} \boldsymbol{\nu}^{k}+\mathbf{F}_{\phi j}^{k l} \boldsymbol{\nu}^{l}+\mathbf{F}_{\phi \phi}^{k k} \boldsymbol{\gamma}^{k}+\mathbf{F}_{\phi \phi}^{k l} \boldsymbol{\gamma}^{l}=0 \\
& \mathbf{M}_{j j}^{k} \boldsymbol{\nu}^{k}+\mathbf{M}_{j \phi}^{k} \boldsymbol{\gamma}^{k}+\mathbf{F}_{j \phi}^{k k} \boldsymbol{\gamma}^{k}+\mathbf{F}_{j \phi}^{k l} \boldsymbol{\gamma}^{l}=\boldsymbol{f}_{\Gamma_{\mathrm{dc}}}^{k}
\end{aligned}
$$

with $f_{\Gamma_{\mathrm{dc}}}^{k}$ rising from the Dirichlet boundary condition, and

$$
\begin{aligned}
& \boldsymbol{\nu}^{k}=\left[\begin{array}{lll}
\boldsymbol{\nu}_{x}^{k} & \boldsymbol{\nu}_{y}^{k} & \boldsymbol{\nu}_{z}^{k}
\end{array}\right]^{T} \\
& \boldsymbol{\nu}^{l}=\left[\begin{array}{lll}
\boldsymbol{\nu}_{x}^{l} & \boldsymbol{\nu}_{y}^{l} & \boldsymbol{\nu}_{z}^{l}
\end{array}\right]^{T} \\
& \mathbf{M}_{\phi j}^{k}=\left[\begin{array}{lll}
\mathbf{M}_{\phi j_{x}}^{k} & \mathbf{M}_{\phi j_{y}}^{k} & \mathbf{M}_{\phi j_{z}}^{k}
\end{array}\right] \\
& \mathbf{M}_{j j}^{k}=\left[\begin{array}{ccc}
\mathbf{M}_{j_{x} j_{x}}^{k} & \mathbf{0} & \mathbf{0} \\
\mathbf{0} & \mathbf{M}_{j_{y} j_{y}}^{k} & \mathbf{0} \\
\mathbf{0} & \mathbf{0} & \mathbf{M}_{j_{z} j_{z}}^{k}
\end{array}\right] \\
& \mathbf{M}_{j \phi}^{k}=\left[\begin{array}{lll}
\mathbf{M}_{j_{x} \phi}^{k} & \mathbf{M}_{j_{y} \phi} & \mathbf{M}_{j_{z} \phi}
\end{array}\right]^{T} \\
& \mathbf{F}_{\phi j}^{k k}=\left[\begin{array}{lll}
\mathbf{F}_{\phi j_{x}}^{k k} & \mathbf{F}_{\phi j_{y}}^{k k} & \mathbf{F}_{\phi j_{z}}^{k k}
\end{array}\right] \\
& \mathbf{F}_{\phi j}^{k l}=\left[\begin{array}{lll}
\mathbf{F}_{\phi j_{x}}^{k l} & \mathbf{F}_{\phi j_{y}}^{k l} & \mathbf{F}_{\phi j_{z}}^{k l}
\end{array}\right] \\
& \mathbf{F}_{j \phi}^{k k}=\left[\begin{array}{lll}
\mathbf{F}_{j_{x} \phi}^{k k} & \mathbf{F}_{j_{y} \phi}^{k k} & \mathbf{F}_{j_{z} \phi}^{k k}
\end{array}\right]^{T} \\
& \mathbf{F}_{j \phi}^{k l}=\left[\begin{array}{lll}
\mathbf{F}_{j_{x} \phi}^{k l} & \mathbf{F}_{j_{y} \phi}^{k l} & \mathbf{F}_{j_{z} \phi}^{k l}
\end{array}\right]^{T}
\end{aligned}
$$

where the general definitions of matrix entries in the above are given by

$$
\begin{aligned}
& {\left[\mathbf{M}_{\phi j_{\xi}}^{k}\right]_{p q}=\int_{\Omega_{k}} \frac{\partial \varphi_{p}^{k}}{\partial_{\xi}} \cdot \psi_{\xi, q}^{k} d V} \\
& {\left[\mathbf{M}_{j_{\xi} j_{\xi}}^{k}\right]_{p q}=\int_{\Omega_{k}} \psi_{\xi, p}^{k} \cdot \psi_{\xi, q}^{k} d V} \\
& {\left[\mathbf{M}_{j_{\xi} \phi}^{k}\right]_{p q}=-\sigma^{k} \int_{\Omega_{k}} \frac{\partial \psi_{\xi, p}^{k}}{\partial_{\xi}} \cdot \varphi_{q}^{k} d V} \\
& {\left[\mathbf{F}_{\phi j_{\xi}}^{k k}\right]_{p q}=-n_{\xi} C_{10} \int_{\Gamma_{k l}} \varphi_{p}^{k} \cdot \psi_{\xi, q}^{k} d S} \\
& {\left[\mathbf{F}_{\phi j_{\xi}}^{k l}\right]_{p q}=-n_{\xi} C_{10} \int_{\Gamma_{k l}} \varphi_{p}^{k} \cdot \psi_{\xi, q}^{l} d S}
\end{aligned}
$$




$$
\begin{aligned}
& {\left[\mathbf{F}_{\phi \phi}^{k k}\right]_{p q}=-C_{12} \int_{\Gamma_{k l}} \varphi_{p}^{k} \cdot \varphi_{q}^{k} d S} \\
& {\left[\mathbf{F}_{\phi \phi}^{k l}\right]_{p q}=C_{12} \int_{\Gamma_{k l}} \varphi_{p}^{k} \cdot \varphi_{q}^{l} d S} \\
& {\left[\mathbf{F}_{j_{\xi} \phi}^{k k}\right]_{p q}=n_{\xi} C_{20} \int_{\Gamma_{k l}} \sigma^{k} \psi_{\xi, p}^{k} \cdot \varphi_{q}^{k} d S} \\
& {\left[\mathbf{F}_{j_{\xi} \phi}^{k l}\right]_{p q}=n_{\xi} C_{20} \int_{\Gamma_{k l}} \sigma^{k} \psi_{\xi, p}^{k} \cdot \varphi_{q}^{l} d S}
\end{aligned}
$$

As the DC current flowing through metal conductors, it will be accompanied with Joule Heating that is evaluated by

$$
Q=\sigma|\mathbf{E}|^{2}
$$

Thus, the temperature of conductors will rise, which consequently alters the resistivity of conductors in terms of the relation given by

$$
\rho(T)=\rho_{0}\left[1+\alpha\left(T-T_{0}\right)\right]
$$

with $\rho_{0}$ denoting the resistivity at temperature $T_{0}$ and $\alpha$ representing the conductor's temperature coefficient.

As a result, to get the DC IR drop with thermal effects included, the thermal equation must be solved simultaneously.

\section{B. Thermal Equation}

Though the temperature is a function of time, only the temperature at the steady-state is more of interest, thus the steady-state thermal equation is going to be solved, which is defined as

$$
\begin{aligned}
& -\nabla \cdot \mathbf{q}+Q=0 \\
& \mathbf{q}=-\kappa \nabla T
\end{aligned}
$$

where $\mathbf{q}$ is the thermal flux, $\kappa$ is the thermal conductivity, $Q$ is the thermal source given in (31), and $\mathbf{q}$ and $T$ are subjected to the boundary conditions given by

$$
\begin{aligned}
& T=T_{0} \quad \text { on } \Pi_{\mathrm{dc}} \\
& \hat{\mathbf{n}} \cdot \mathbf{q}=-h\left(T-T_{a}\right) \text { on } \Pi_{\mathrm{c}}
\end{aligned}
$$

with $T_{a}$ denoting the ambient temperature and $h$ representing the convection coefficient.

Similar to the DG formulation process for the electrical equation, the Galerkin test is applied to (33) and (34)

$$
\begin{aligned}
& \int_{\Omega_{k}} \nabla \breve{\varphi}_{p}^{k} \cdot \mathbf{q}^{k} d V=\int_{\Omega_{k}} \breve{\varphi}_{p}^{k} \cdot Q^{k} d V+\int_{\Gamma_{k l}} \breve{\varphi}_{p}^{k}\left(\hat{\mathbf{n}} \cdot \tilde{\mathbf{q}}^{k}\right) d S \\
& \int_{\Omega_{k}} \breve{\psi}_{\xi, q}^{k} \cdot q_{\xi}^{k} d V=\int_{\Omega_{k}} \kappa^{k} \partial_{\xi} \breve{\psi}_{q}^{k} \cdot T^{k} d V-n_{\xi} \int_{\Gamma_{k l}} \kappa^{k} \psi_{\xi, q}^{k} \cdot \tilde{T}^{k} d S
\end{aligned}
$$

where $\breve{\varphi}_{p}^{k}$ and $\breve{\psi}_{\xi, q}^{k}$ are the $p$-th and the $q$-th nodal basis functions for $T$ and $q_{\xi}(\xi=x, y, z)$ in the $k$-th subdomain, respectively; the other two terms $\tilde{\mathbf{q}}^{k}$ and $\tilde{T}^{k}$ are termed as numerical flux defined by [15]-[17]

$$
\begin{aligned}
& \hat{\mathbf{n}} \cdot \tilde{\mathbf{q}}^{k}=\check{C}_{10}\left(\hat{\mathbf{n}} \cdot \mathbf{q}^{k}+\hat{\mathbf{n}} \cdot \mathbf{q}^{l}\right)+\check{C}_{11}\left(\hat{\mathbf{n}} \cdot \mathbf{q}^{k}-\hat{\mathbf{n}} \cdot \mathbf{q}^{l}\right) \\
& +\check{C}_{12}\left(T^{k}-T^{l}\right) \\
& \tilde{T}^{k}=\check{C}_{20}\left(T^{k}+T^{l}\right)+\check{C}_{21}\left(\hat{\mathbf{n}} \cdot \mathbf{q}^{k}-\hat{\mathbf{n}} \cdot \mathbf{q}^{l}\right) \\
& +\check{C}_{22}\left(T^{k}-T^{l}\right)
\end{aligned}
$$

In this work, the upwind flux is employed [15], i.e., $\check{C}_{10}=0.5$, $\check{C}_{11}=0, \check{C}_{12}=-4$, and $\check{C}_{20}=0.5, \check{C}_{21}=0, \check{C}_{22}=0$.

Next, substitute (39) and (40) into (37) and (38), and further expand the unknowns by the nodal basis functions: $T^{k}=\sum_{n=1}^{N_{k}} \lambda_{n}^{k} \breve{\varphi}_{n}^{k}$ and $q_{\xi}^{k}=\sum_{m=1}^{M_{\xi}^{k}} \chi_{\xi, m}^{k} \breve{\psi}_{\xi, m}^{k}$ with $\lambda_{n}^{k}$ and $\chi_{m}^{k}$ representing the unknown expansion coefficients, the following matrix equations can be reached after a few mathematical manipulations,

$$
\begin{aligned}
& \mathbf{M}_{T q}^{k} \chi^{k}+\mathbf{F}_{T q}^{k k} \chi^{k}+\mathbf{F}_{T q}^{k l} \chi^{l}+\mathbf{F}_{T T}^{k k} \boldsymbol{\lambda}^{k}+\mathbf{F}_{T T}^{k l} \boldsymbol{\lambda}^{l}=\boldsymbol{f}_{\Pi_{\mathrm{c}}}^{k} \\
& \mathbf{M}_{q q}^{k} \chi^{k}+\mathbf{M}_{q T}^{k} \boldsymbol{\lambda}^{k}+\mathbf{F}_{q T}^{k k} \boldsymbol{\lambda}^{k}+\mathbf{F}_{q T}^{k l} \boldsymbol{\lambda}^{l}=\boldsymbol{f}_{\Pi_{\mathrm{dc}}}^{k}
\end{aligned}
$$

where the definitions of matrices as well as the corresponding entries involved in (41) and (42) can be conveniently derived by permuting the basis functions and related material parameters given in the electrical equations from (11) to (30).

\section{Electrical-Thermal Co-analysis}

Due to the temperature dependent electric conductivity, the electrical matrix equations (11) and (12) couple with the thermal equations (41) and (42), resulting in a nonlinear matrix system. To solve it accurately and efficiently, the electrical and the thermal equations will be iteratively solved using the fixed point method. The pesudocode of the solving scheme is shown as below:

Intializing: $\rho=\rho_{0}, T=T_{0}$, the stop criterion $\zeta_{0}$, and the number of iterations $N_{i}$

For $i=1: N_{i}$ do

Step 1 : Solve the electrical equation

Step 2 : Calculate Joule Heating

Step 3 : Solve the thermal equation

Step 4 : Update the resistivity

Step 5 : Calculate $L_{2}$ relative norm error $\delta \rho$

If $\delta \rho<\zeta_{0}$ then

Output solutions of interests

Exit

\section{End If}

End For

Step $6: i=i+1$

\section{Block Thomas Method}

Due to the implicit form of the numerical flux, the established matrix equations in (11), (12), (41) and (42) will couple with matrix equations in adjacent subdomains, which results in a huge matrix system, e.g., the dimension of the electrical 
matrix equation is around $\sum_{k=1}^{K}\left(P^{k}+Q_{x}^{k}+Q_{y}^{k}+Q_{z}^{k}\right)$ by $\sum_{k=1}^{K}\left(P^{k}+Q_{x}^{k}+Q_{y}^{k}+Q_{z}^{k}\right)$, and the dimension of the thermal matrix equation is around $\sum_{k=1}^{K}\left(N^{k}+M_{x}^{k}+M_{y}^{k}+M_{z}^{k}\right)$ by $\sum_{k=1}^{K}\left(N^{k}+M_{x}^{k}+M_{y}^{k}+M_{z}^{k}\right)$.

To solve the whole matrix system in a subdomain by subdomain scheme, either the block Gauss-Seidel method or the block Thomas method can be referred. The block GaussSeidel method is an iterative method and thus its convergence cannot be guaranteed if the formulated matrix equation is not symmetrical positive definite or diagonally dominant. Instead, the block Thomas method is a direct solver [18], thereby it is free of convergence problem. To implement the block Thomas method, the teared subdomains are arranged in a nested sequence, which results in a serial coupling scheme. As a result, a block tridiagonal matrix pattern is formed.

To have an intuitive insight into the tridiagonal matrix system formulation, the matrix equations (11) and (12) for the electrical potential are rewritten as a compact form,

$$
\mathbf{M}^{k} \boldsymbol{v}^{k}+\mathbf{F}^{k l} \boldsymbol{v}^{l}=\boldsymbol{f}^{k}
$$

with

$$
\begin{aligned}
& \boldsymbol{v}^{k, l}=\left[\begin{array}{l}
\boldsymbol{\nu}^{k, l} \\
\boldsymbol{\gamma}^{k, l}
\end{array}\right] \\
& \boldsymbol{f}^{k}=\left[\begin{array}{c}
\mathbf{0} \\
\boldsymbol{f}_{\Gamma_{\mathrm{dc}}}^{k}
\end{array}\right] \\
& \mathbf{M}^{k}=\left[\begin{array}{cc}
\mathbf{M}_{\phi j}^{k}+\mathbf{F}_{\phi j}^{k k} & \mathbf{F}_{\phi \phi}^{k k} \\
\mathbf{M}_{j j}^{k} & \mathbf{M}_{j \phi}^{k}+\mathbf{F}_{j \phi}^{k k}
\end{array}\right] \\
& \mathbf{F}^{k l}=\left[\begin{array}{cc}
\mathbf{F}_{\phi j}^{k l} & \mathbf{F}_{\phi \phi}^{k l} \\
\mathbf{0} & \mathbf{F}_{\phi \phi}^{k l}
\end{array}\right]
\end{aligned}
$$

Because of the nested subdomains, the whole matrix system can be written as a block tridiagonal one, that is,

$$
\left[\begin{array}{ccccc}
\mathbf{M}^{1} & \mathbf{F}^{12} & \mathbf{0} & \cdots & \mathbf{0} \\
\mathbf{F}^{21} & \mathbf{M}^{2} & \mathbf{F}^{23} & \cdots & \mathbf{0} \\
\mathbf{0} & \mathbf{F}^{32} & \mathbf{M}^{3} & \cdots & \mathbf{0} \\
\vdots & \vdots & \vdots & \ddots & \vdots \\
\mathbf{0} & \mathbf{0} & \cdots & \mathbf{F}^{K, K-1} & \mathbf{M}^{K}
\end{array}\right]\left[\begin{array}{c}
v^{1} \\
v^{2} \\
v^{3} \\
\vdots \\
v^{K}
\end{array}\right]=\left[\begin{array}{c}
\boldsymbol{f}^{1} \\
\boldsymbol{f}^{2} \\
\boldsymbol{f}^{3} \\
\vdots \\
\boldsymbol{f}^{K}
\end{array}\right]
$$

To proceed the block Thomas method, the matrix on the left hand side should be written as the production between a lower and a upper block tridiagonal matrix in terms of LU decomposition, namely,

$$
\left[\begin{array}{ccccc}
\mathbf{M}^{1} & \mathbf{F}^{12} & \mathbf{0} & \cdots & \mathbf{0} \\
\mathbf{F}^{21} & \mathbf{M}^{2} & \mathbf{F}^{23} & \cdots & \mathbf{0} \\
\mathbf{0} & \mathbf{F}^{32} & \mathbf{M}^{3} & \cdots & \mathbf{0} \\
\vdots & \vdots & \vdots & \ddots & \vdots \\
\mathbf{0} & \mathbf{0} & \cdots & \mathbf{F}^{K, K-1} & \mathbf{M}^{K}
\end{array}\right]=\mathbf{L}_{B} \mathbf{U}_{B}
$$

with

$$
\begin{aligned}
\mathbf{U}_{B} & =\left[\begin{array}{cccccc}
\mathbf{I} & \widetilde{\mathbf{F}}^{12} & \mathbf{0} & \mathbf{0} & \cdots & \mathbf{0} \\
\mathbf{0} & \mathbf{I} & \widetilde{\mathbf{F}}^{23} & \mathbf{0} & \cdots & \mathbf{0} \\
\mathbf{0} & \mathbf{0} & \mathbf{I} & \widetilde{\mathbf{F}}^{34} & \cdots & \mathbf{0} \\
\vdots & \vdots & \vdots & \vdots & \ddots & \vdots \\
\mathbf{0} & \cdots & \mathbf{0} & \mathbf{0} & \mathbf{I} & \widetilde{\mathbf{F}}^{K-1, K} \\
\mathbf{0} & \cdots & \mathbf{0} & \mathbf{0} & \mathbf{0} & \mathbf{I}
\end{array}\right] \\
\mathbf{L}_{B} & =\left[\begin{array}{cccccc}
\widetilde{\mathbf{M}}^{1} & \mathbf{0} & \mathbf{0} & \cdots & \mathbf{0} & \mathbf{0} \\
\mathbf{F}^{21} & \widetilde{\mathbf{M}}^{2} & \mathbf{0} & \cdots & \mathbf{0} & \mathbf{0} \\
\mathbf{0} & \mathbf{F}^{32} & \widetilde{\mathbf{M}}^{3} & \cdots & \mathbf{0} & \mathbf{0} \\
\vdots & \vdots & \vdots & \ddots & \vdots & \vdots \\
\mathbf{0} & \cdots & \mathbf{0} & \mathbf{F}^{K-1, K-2} & \widetilde{\mathbf{M}}^{K-1} & \mathbf{0} \\
\mathbf{0} & \cdots & \mathbf{0} & \mathbf{0} & \mathbf{F}^{K, K-1} & \widetilde{\mathbf{M}}^{K}
\end{array}\right]
\end{aligned}
$$

where

$$
\widetilde{\mathbf{M}}^{1}=\mathbf{M}^{1}
$$

$$
\begin{aligned}
& \widetilde{\mathbf{M}}^{k}=\mathbf{M}^{k}-\mathbf{F}^{k, k-1} \widetilde{\mathbf{F}}^{k-1, k}, \quad k=2, \cdots, K \\
& \widetilde{\mathbf{F}}^{k, k+1}=\left(\widetilde{\mathbf{M}}^{k}\right)^{-1} \mathbf{F}^{k, k+1}, \quad k=1, \cdots, K-1
\end{aligned}
$$

Instead of solving (48) straightforwardly, the solution $v$ can be obtained in two steps: i) solving $\mathbf{L}_{\mathbf{B}} \tilde{\boldsymbol{v}}=\boldsymbol{f}$ firstly; ii) then solving $\mathrm{U}_{\mathrm{B}} \boldsymbol{v}=\tilde{\boldsymbol{v}}$. The pseudocode of the block Thomas method is provided as below:

Solving Scheme: Block Thomas Method

$$
\begin{aligned}
& \text { Solve } \widetilde{\mathbf{F}}^{12}: \widetilde{\mathbf{F}}^{12}=\left(\mathbf{M}^{1}\right)^{-1} \mathbf{F}^{12} \\
& \text { For } k=2: K-1 \text { do } \\
& \quad \widetilde{\mathbf{M}}^{k}=\mathbf{M}^{k}-\mathbf{F}^{k, k-1} \widetilde{\mathbf{F}}^{k-1, k} \\
& \quad \text { Solve } \widetilde{\mathbf{F}}^{k, k+1}: \widetilde{\mathbf{F}}^{k, k+1}=\left(\widetilde{\mathbf{M}}^{k}\right)^{-1} \mathbf{F}^{k, k+1} \\
& \text { End For } \\
& \widetilde{\mathbf{M}}^{K}=\mathbf{M}^{K}-\mathbf{F}^{K, K-1} \widetilde{\mathbf{F}}^{K-1, K} \\
& \text { Solve } \widetilde{\boldsymbol{v}}^{1}: \widetilde{\boldsymbol{v}}^{1}=\left(\mathbf{M}^{1}\right)^{-1} \boldsymbol{f}^{1} \\
& \text { For } k=2: K \text { do } \\
& \quad \text { Solve } \widetilde{\boldsymbol{v}}^{k}: \widetilde{\boldsymbol{v}}^{k}=\left(\widetilde{\mathbf{M}}^{k}\right)^{-1}\left(\boldsymbol{f}^{k}-\mathbf{F}^{k, k-1} \widetilde{\boldsymbol{v}}^{k-1}\right) \\
& \text { End For } \\
& \boldsymbol{v}^{K}=\widetilde{\boldsymbol{v}}^{K} \\
& \text { For } k=K-1:-1: 1 \text { do } \\
& \quad \boldsymbol{v}^{k}=\widetilde{v}^{k}-\widetilde{\mathbf{F}}^{k, k+1} \boldsymbol{v}^{k+1}
\end{aligned}
$$$$
\text { End For }
$$

It is noted that the whole domain is solved by a subdomain by subdomain strategy, thus dramatically decreasing the dimension of the matrix equation, i.e., the dimension of the matrix equation to be solved now is $\left(P^{k}+Q_{x}^{k}+Q_{y}^{k}+Q_{z}^{k}\right)$ by $\left(P^{k}+Q_{x}^{k}+Q_{y}^{k}+Q_{z}^{k}\right)$, which no doubt significantly lowers the computational request. 


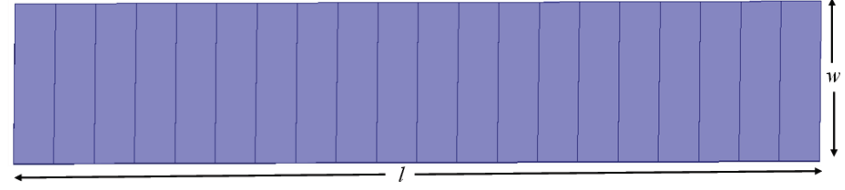

Fig. 1. Geometry information of the power plane. The length $l$, the width $w$, and the thickness $t$ are $27 \mathrm{~mm}, 6 \mathrm{~mm}$ and $0.05 \mathrm{~mm}$, respectively.

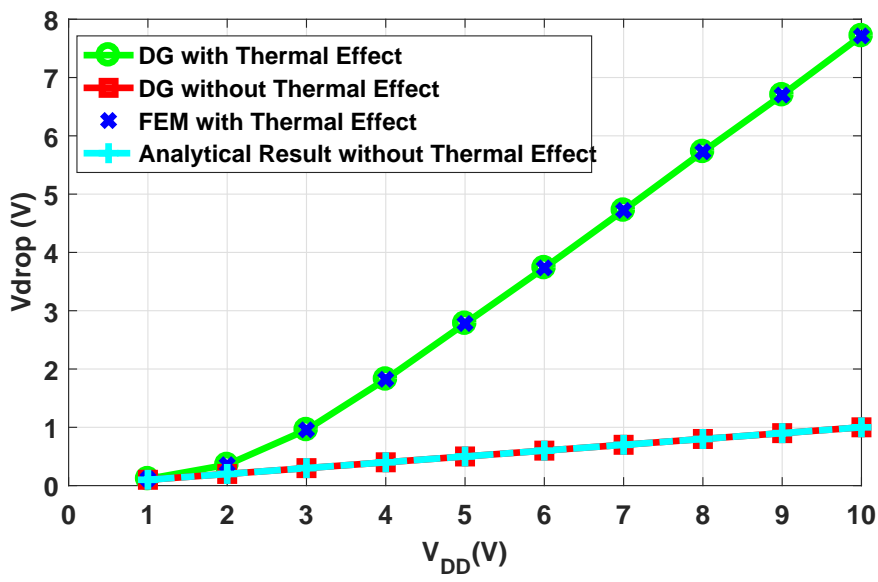

Fig. 2. The calculated voltage drop when different $V_{\mathrm{DD}}$ is attached.

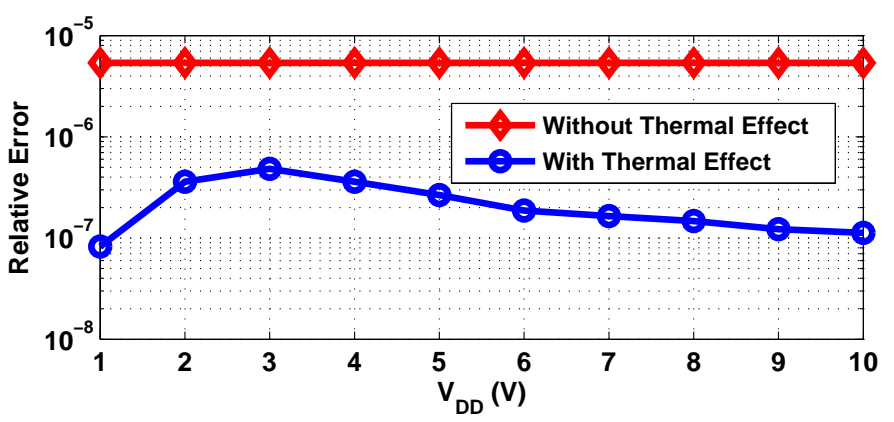

Fig. 3. The relative difference for voltage drops calculated by the proposed algorithm and the corresponding reference method.

The above formulations are also valid for the thermal matrix equations in (41) and (42), the details will not be repeated here due to the limited space.

\section{NUMERICAL RESULTS}

In this part, the proposed algorithm is validated by several representative examples, where the accuracy and the capability on modeling complex geometries are benchmarked. For all examples, the first-order nodal basis functions are used, i.e., the basis functions are located at four vertices of each tetrahedron. In addition, the ground planes in the following examples are virtual ones, and the electric potential on them are assumed to be zero.

\section{A. A Thin Power Plane}

With the aim to verify the accuracy of the proposed algorith$\mathrm{m}$, a thin metal plane shown in Fig. 1 is investigated. A voltage source $V_{\mathrm{DD}}$ is placed at the left end surface of the plane, while the right end surface of the plane is attached to a resistive load

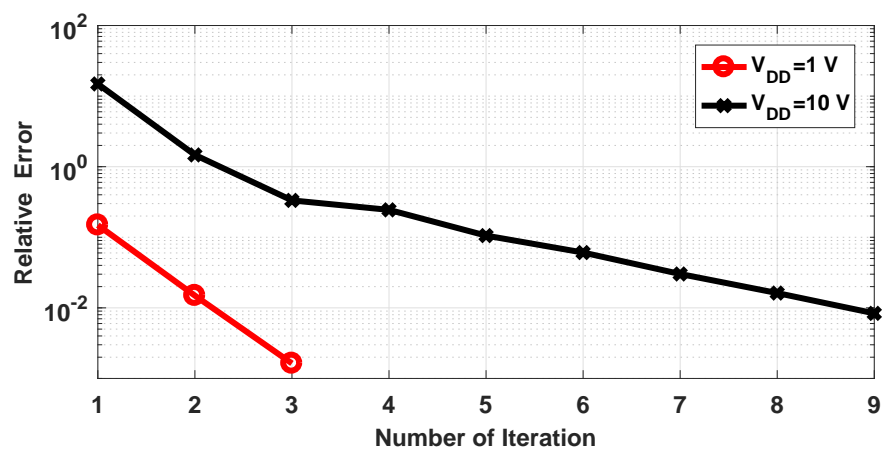

Fig. 4. Convergence properties pertinent to different DC supplies.

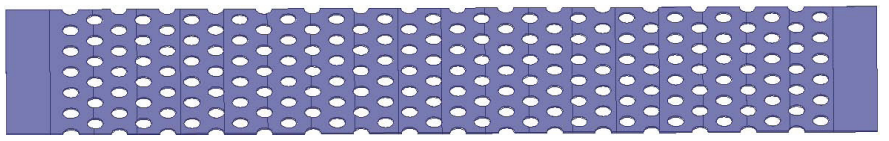

Fig. 5. "Swiss cheese" effect: large number of holes are present on the power plane. The dimension and material property of the plane are the same as those in the first example, the radius of the via hole is $0.508 \mathrm{~mm}$, and the separation distances between two neighboring via holes are $1 \mathrm{~mm}$ along the width direction and $0.75 \mathrm{~mm}$ along the length direction.

with $R_{L}=0.01458 \Omega$. In addition, the convection boundary condition is applied at the top and the bottom surfaces with convection coefficient $h=500 \mathrm{~W} /\left(\mathrm{m}^{2} \cdot \mathrm{K}\right)$. The resistivity $\rho_{0}$, the thermal conductivity $\kappa$, the temperature coefficient $\alpha$, and the ambient temperature $T_{a}$ are set as $1.8 \times 10^{-8} \Omega \mathrm{m}, 400$ $\mathrm{W} /(\mathrm{m} \cdot \mathrm{K}), 0.0039 \mathrm{~K}^{-1}$ and $300 \mathrm{~K}$, respectively. The plane is split into 20 subdomains along the length direction, which results in 92,410 and 92,500 unknowns for the electrical and the thermal matrix system, respectively.

The accuracy of the proposed DG algorithm is validated by comparing with those obtained from FEM simulation. As shown in Fig. 2, the results are consistent with each other excellently. Moreover, the results without thermal effects are also given and compared with the analytical results calculated by $V_{\text {drop }}=V_{\mathrm{DD}} \frac{R}{R+R_{L}}$ with $R=\rho_{0} l / S$ and $S$ denoting the area of the cross-section. The relative error between the proposed algorithm and the reference solution is also plotted in Fig. 3.

To have a basic insight into the convergence rate of the iterative scheme, the number of iterations to reach the intended tolerance is shown in Fig. 4. As can be seen, it requires more iterations with the increase of $V_{\mathrm{DD}}$, which is due to that more Joule Heating is generated and thus alters the resistivity of the conductor more dramatically. The corresponding CPU time and the memory consumption $\left(V_{\mathrm{DD}}=10 \mathrm{~V}\right.$ case $)$ for the proposed DG method are around 3 minutes and $600 \mathrm{M}$, while FEM takes around 10 minutes with memory cost less than 100 M. As can be seen, although the proposed algorithm has better CPU efficiency, but its memory cost is also higher than FEM, which is due to that the matrix $\tilde{\mathbf{F}}$ in (50) is no longer a sparse one.

\section{B. Swiss Cheese Effect}

A large number of holes are formed around vias passing through the power planes, this "Swiss cheese" effect, as shown 


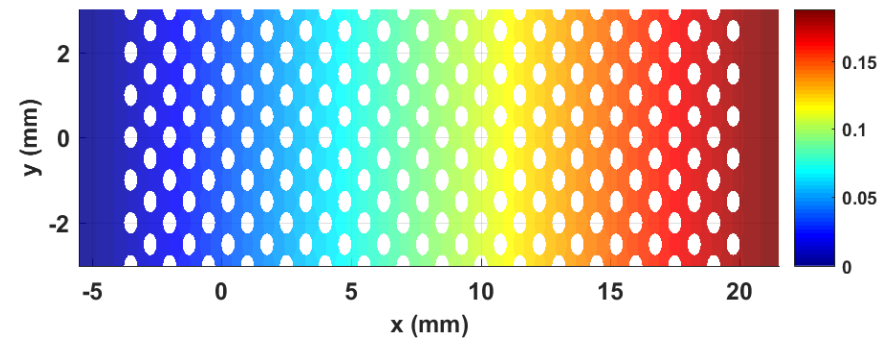

(a)

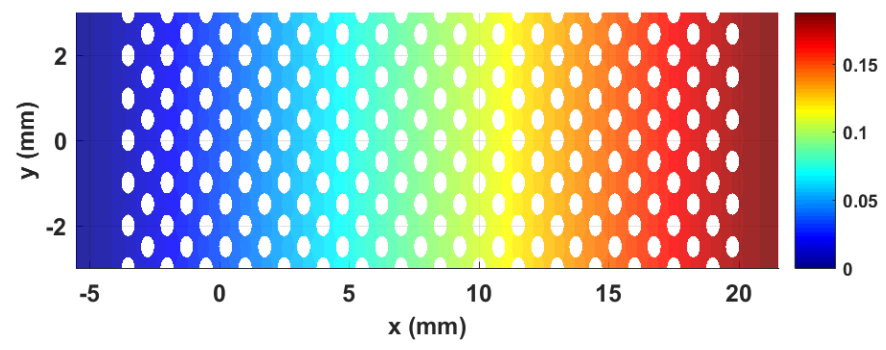

(b)

Fig. 6. The voltage drop (units: V) of the power plane in the presence of "Swiss cheese" effect. (a) Results by DG method based electrical-thermal co-simulation, (b) Results by FEM based electrical-thermal co-simulation.

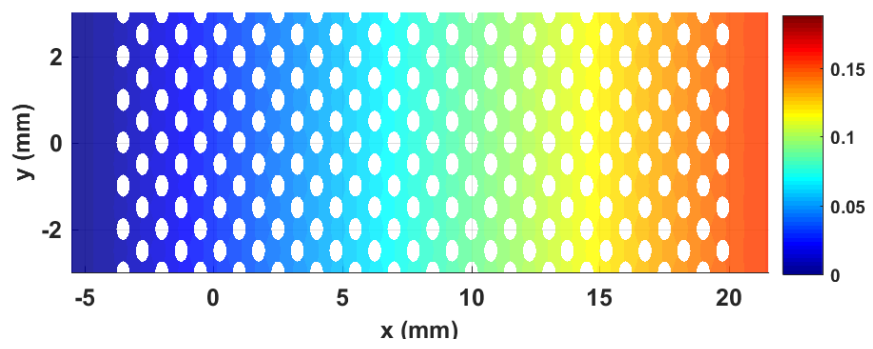

Fig. 7. The voltage drop (units: V) of the power plane in the presence of "Swiss cheese" effect calculated by DG method without considering thermal effect.

in Fig. 5, can seriously deteriorate the PI performance of the power plane. The narrower space between adjacent voids significantly increases the current density, thus resulting in large amount of Joule Heating. Consequently, the impedance of the power plane becomes larger as well, which no doubt is detrimental to the DC IR-drop performance.

To verify the above statement, a voltage source with $V_{\mathrm{DD}}=1 \mathrm{~V}$ is attached to the left end surface, while the right end is connected to a resistive load with $R_{L}=0.01458 \Omega$; the convection boundary condition is applied simultaneously to the top and the bottom surface with convection coefficient $h=500 \mathrm{~W} /\left(\mathrm{m}^{2} \cdot \mathrm{K}\right)$. The whole plane is teared into 20 subdomains along the length direction, resulting in 135, 094 unknowns for the electrical equation and 135,216 unknowns for the thermal equation. Firstly, the voltage drop is characterized based on the proposed electrical-thermal co-simulation algorithm, as shown in Fig. 6. For accuracy validation, the reference result calculated by FEM is also given. Excellent consistency is obviously noted. To have a better insight into the impact of thermal effect on the resistance, the voltage drop excluding thermal effect is also calculated by the proposed DG

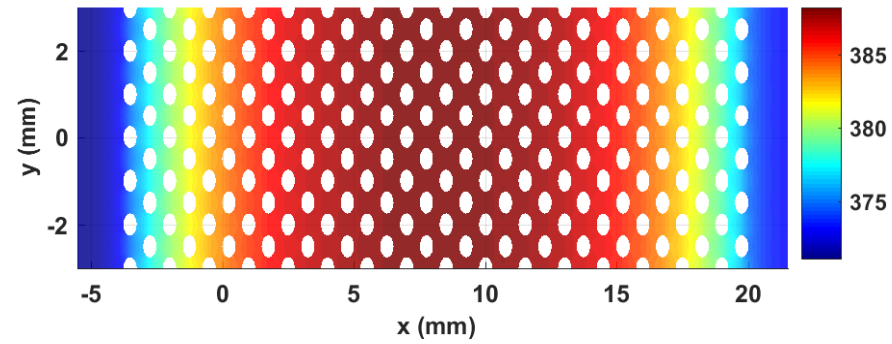

(a)

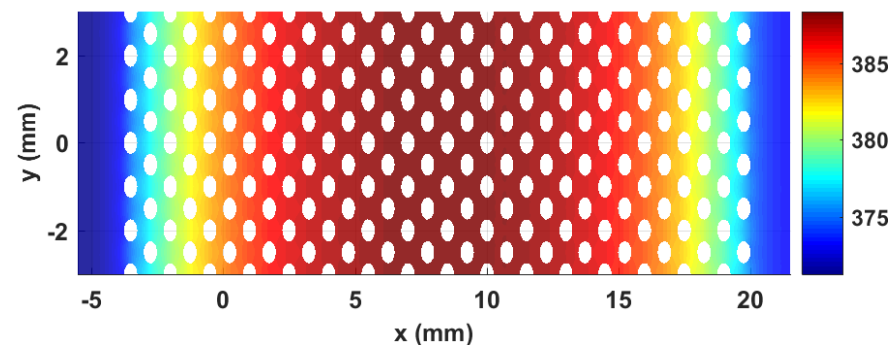

(b)

Fig. 8. Temperature profile (units: K) of the power plane in the presence of "Swiss cheese" effect. (a) DG method, (b) FEM.

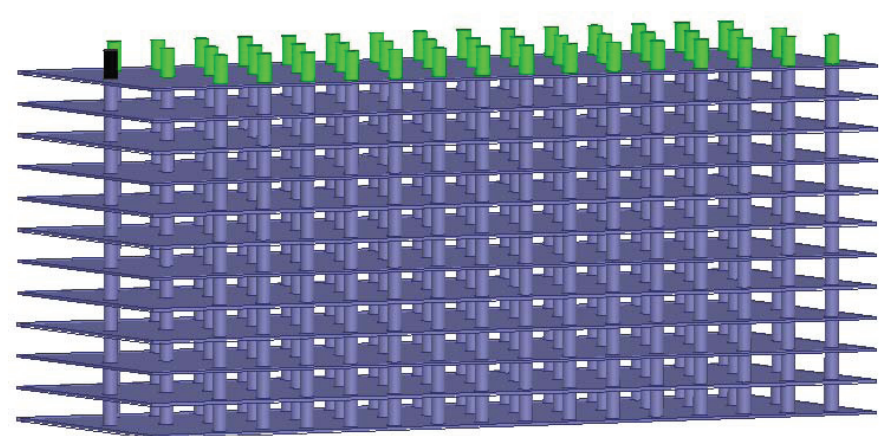

Fig. 9. Geometrical profile of a 3D power planes having 12 layers stacked vertically.

method with result shown in Fig. 7. The maximum voltage drop considering thermal effect is increased from $0.1528 \mathrm{~V}$ to $0.1876 \mathrm{~V}$, i.e., $23 \%$ more voltage drop is sacrificed due to Joule Heating. Secondly, the temperature profile of the power plane obtained from the proposed DG algorithm is also presented in Fig. 8. As can been seen, the temperature in the proximity of holes are obviously higher. The maximum temperature is around $388 \mathrm{~K}$, while the maximum temperature without "Swiss cheese" effect obtained in the first example is around $343 \mathrm{~K}$, which further reveals the negative impact of "Swiss cheese" effect. With regarding to the accuracy, the simulation results by FEM is also provided for verification. Again, very good agreements are achieved. For this example, the CPU time and the memory cost of the proposed algorithm are around 4 minutes and $800 \mathrm{M}$, respectively, while FEM takes about 12 minutes with memory cost less than $100 \mathrm{M}$. 


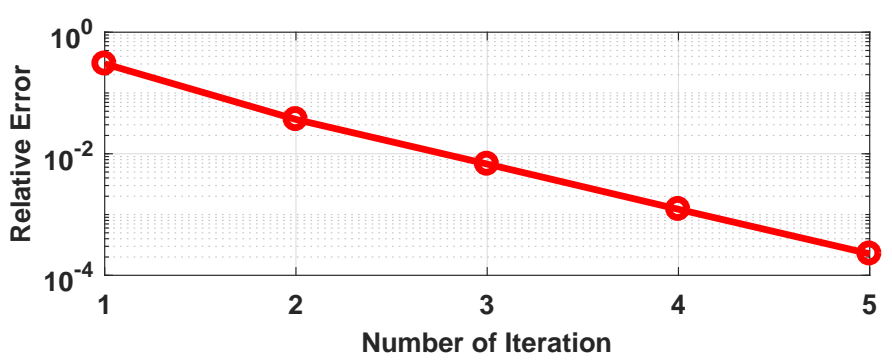

Fig. 10. The convergence property of the iterative electrical-thermal cosimulation scheme for the third example.

\section{A PDN Composed of Twelve Vertically-Stacked Power Planes}

In the third example, a 3D PDN with 12 layer power planes is investigated. As shown in Fig. 9, the vertically stacked power planes are chained by vias in gray color. The length, the width, and the thickness of each power plane are $14.5 \mathrm{~mm}$, $5.08 \mathrm{~mm}$, and $0.0508 \mathrm{~mm}$, respectively; the radius and the height of vias are $0.127 \mathrm{~mm}$ and $0.508 \mathrm{~mm}$, respectively. The material properties are the same as those in the first example. The voltage source $V_{\mathrm{DD}}=1 \mathrm{~V}$ is placed over the top surface of the black via, the green vias are connected with resistive loads with $R_{L}=0.5 \Omega$, and the air convection boundary with $h=500 \mathrm{~W} /\left(\mathrm{m}^{2} \cdot \mathrm{K}\right)$ is placed over the bottom surface of power plane in the first layer. The entire domain is tesselated into 24 subdomains along the vertical direction in a nested sequence, which produces 721,783 unknowns for the electrical equation and 721,796 unknowns for the thermal equation. The electrical-thermal co-simulation takes 5 iterations to reach the predefined stop criterion $\delta \rho=10^{-3}$ with convergence scheme shown in Fig. 10. The corresponding CPU time is around 4 hours and 20 minutes, while the memory cost is about $12 \mathrm{G}$. The 3D potential distribution as well as the 3D temperature profile are plotted in Fig. 11 and Fig. 12, respectively. It is noted that the temperature of the via attached to the voltage source is obviously higher than others due to the higher current density, and the maximum IR-drop is $45.6 \mathrm{mV}$ while the IRdrop without thermal effect is $33.5 \mathrm{mV}$,.i.e., resulting in $36.1 \%$ more voltage loss.

\section{A PDN Composed of Fifteen Layers of Vertically-Stacked Power Planes}

As the last example, a global PDN for a 3D integrated circuit is studied, in which fifteen layers of power planes are vertically stacked along the thickness direction, and a number of resistive loads are placed at each power plane, as shown in Fig. 13, in which the red vias are power vias used to chain the power planes together, while the green vias are signal vias connecting to the $\mathrm{CPU}$ or the memory dies. The length, the width, and the thickness of each power plane are $13.05 \mathrm{~mm}, 7.08 \mathrm{~mm}$, and $0.0508 \mathrm{~mm}$, respectively; the radius and the height of power vias are $0.127 \mathrm{~mm}$ and $0.508 \mathrm{~mm}$, while the radius and the height of the signal vias are $0.127 \mathrm{~mm}$ and $0.381 \mathrm{~mm}$. To consider the load effects of dies, all signal vias are terminated by five parallel connected resistive loads with $R_{L}=50 \Omega$. To analyze the DC-IR drop,

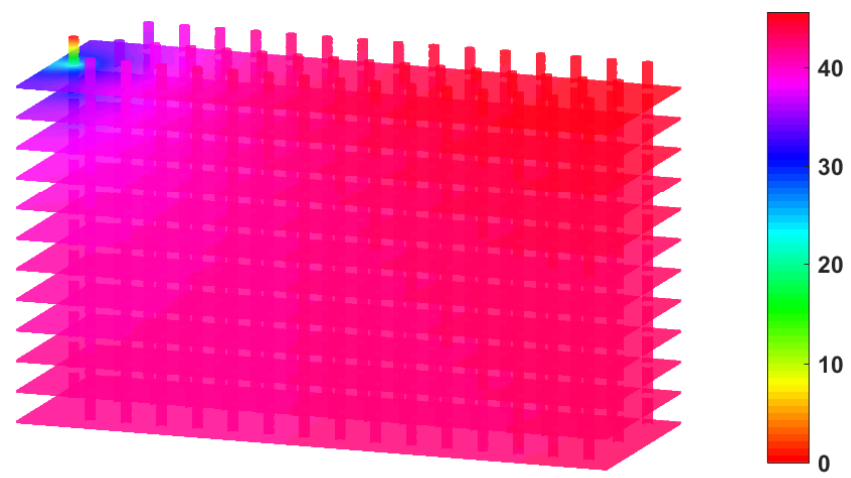

Fig. 11. The voltage drop (units: $\mathrm{mV}$ ) calculated by the proposed DG algorithm

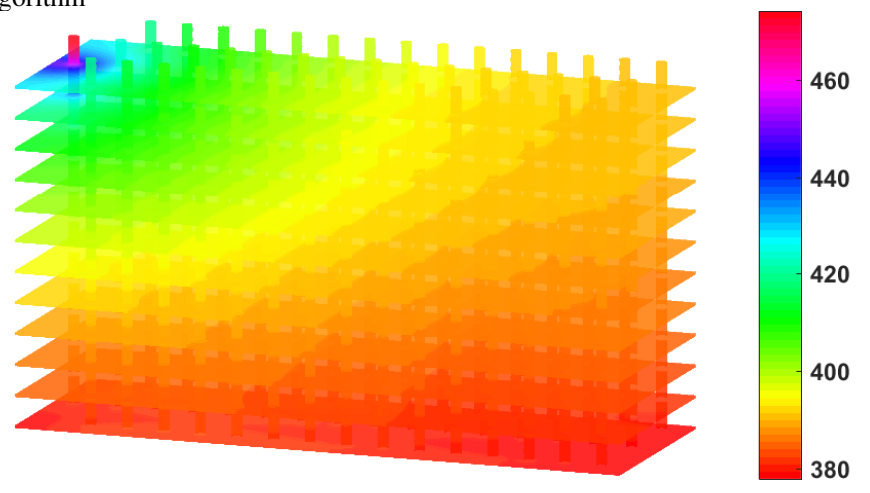

Fig. 12. The temperature profile (units: K) calculated by the proposed DG algorithm

a $1 \mathrm{~V}$ voltage source used as the DC supply is attached to the top surface of the black via, and the air convection cooling with convection coefficient $=100 \mathrm{~W} /\left(\mathrm{m}^{2} \cdot \mathrm{K}\right)$ is placed over the bottom surface of the power plane in the first layer (bottom layer). The whole structure is decomposed into 30 subdomains along the thickness direction, which introduces 862,326 unknowns for the electrical equation and 862,336 unknowns for the thermal equation, but the maximum dimension of the subdomain matrix system is 44,612 by 44,612 . In Fig. 14 and Fig. 15, the calculated voltage drop as well as the temperature distribution are shown, where the maximum IRdrop is $18.1 \mathrm{mV}$ (the IR-drop without thermal effect is 14.8 $\mathrm{mV}, 22.3 \%$ more loss is introduced due to Joule Heating) and the lowest and the highest temperature are increased to $354 \mathrm{~K}$ and $379 \mathrm{~K}$, respectively. The required iteration number with desired accuracy $\sigma_{\rho}=10^{-4}$ is 5 , and the convergence scheme is shown in Fig. 16. The corresponding CPU time for the proposed DG method is about 6 hours and 10 minutes, and the memory cost is around $15 \mathrm{G}$.

\section{CONCLUSION}

A DG method is developed in this work to investigate the DC IR-drop of integrated circuits, where the impact of thermal effects due to Joule Heating is included. The temperature dependent material property results in a nonlinear matrix system, which is iteratively solved by the fixed point method. Due to the implicit numerical flux, the unknowns couple 


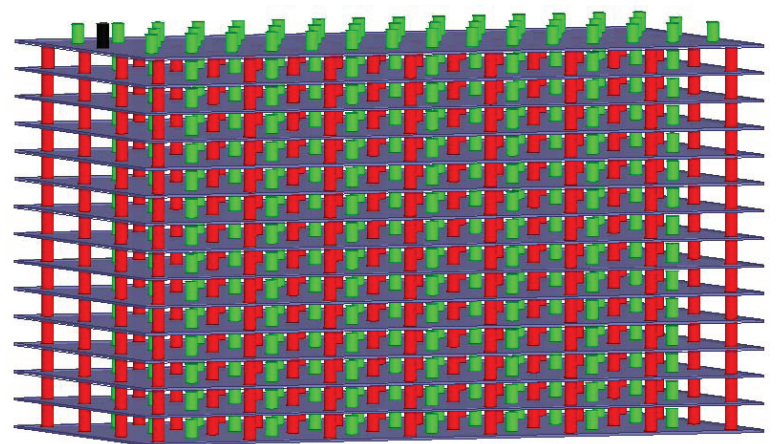

Fig. 13. Illustration of the 3D PDN, where the red vias are named as power vias used for DC supply and the green vias are called signal vias connecting to the active modules such as CPU or memory dies.

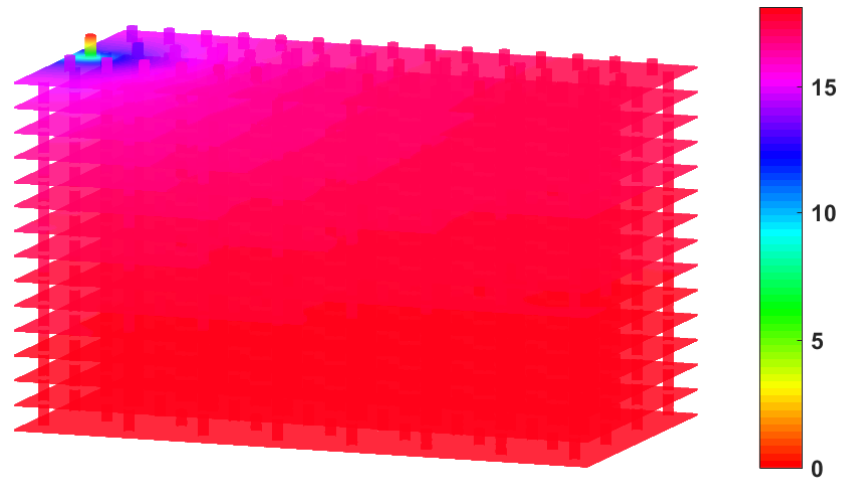

Fig. 14. The voltage drop (units: $\mathrm{mV}$ ) calculated by the proposed DG algorithm

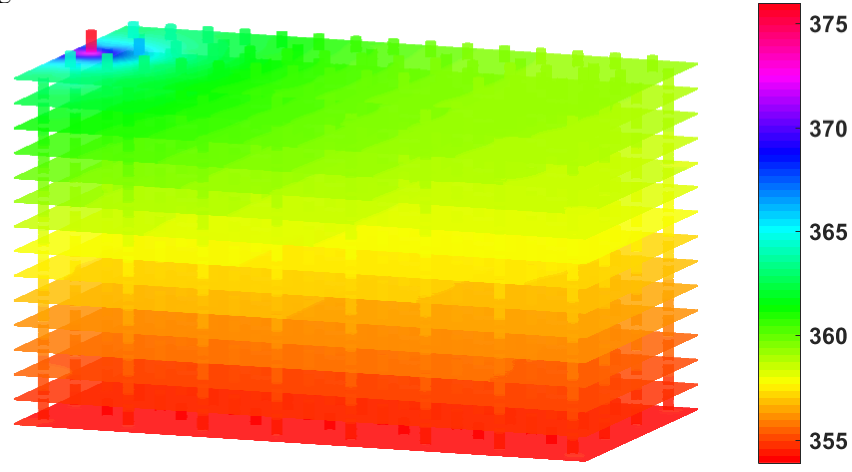

Fig. 15. The temperature profile (units: K) calculated by the proposed DG algorithm

with each other in different subdomains, which causes a huge matrix system. To keep the advantage of DG in solving the entire domain in a subdomain by subdomain scheme, the block Thomas method is resorted, which is a direct solver thus it is free of convergence issues. The proposed approach is benchmarked by four examples by way of comparing with FEM reference and/or analytical solutions.

\section{REFERENCES}

[1] M. S. Zhang and J. Fan, "IR-drop modeling and reduction for highperformance printed circuit boards," IEEE Electromagn. Mag., vol. 4, no. 4, pp. 90-101, 2015.

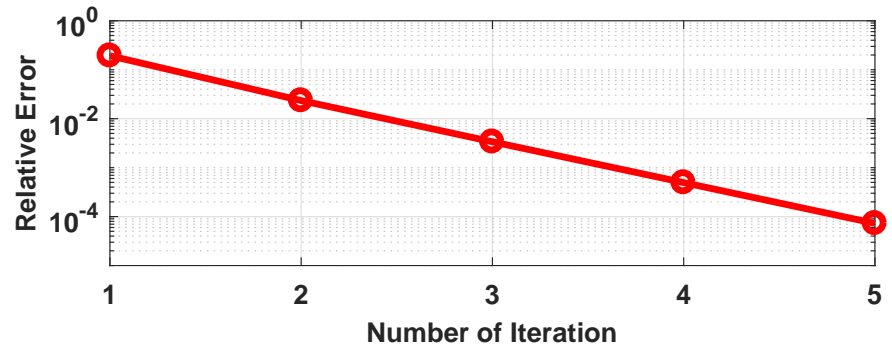

Fig. 16. The convergence property of the iterative electrical-thermal cosimulation scheme for the fourth example.

[2] T.-L. Wu, F. Buesink, and F. Canavero, "Overview of signal integrity and EMC design technologies on PCB: fundamentals and latest progress," IEEE Trans. Electromagn. Compat., vol. 55, no. 4, pp. 624-638, Aug. 2013.

[3] M. Swaminathan, D. Chung, S. G. Talocia, K. Bharath, V. Laddha, and J. Xie, "Designing and modeling for power integrity," IEEE Trans. Electromagn. Compat., vol. 53, no. 2, pp. 288C310, May 2010.

[4] K. Shakeri and J. D. Meindl, "Compact physical IR-drop models for chip/package co-design of gigascale integration (GSI)," IEEE Trans. Electron Devices, vol. 52, no. 6, pp. 1087C1096, Jun. 2005.

[5] J. Xie and M. Swaminathan, "Electrical-thermal co-simulation of 3D integrated systems with micro-fluidic cooling and Joule heating effects," IEEE Trans. Compon., Packag. Manuf. Technol., vol. 1, no. 2, pp. 234C246, Feb. 2011.

[6] Y. Shao, Z. Peng and J. F. Lee, "Thermal-aware DC IR-drop co-analysis using non-conformal domain decomposition methods," Proc. R. Soc. A, vol. 468, pp. 1652-1675, 2012.

[7] T. J. Lu and J. M. Jin, "Electrical-thermal co-cimulation for DC IR-drop analysis of large-scale power delivery," IEEE Trans. Compon., Packag. Manuf. Technol., vo. 4, no. 2, pp. 323-331, Feb. 2014.

[8] T. Lu and J. M. Jin, "Transient electrical-thermal analysis of 3-D power distribution network with FETI-enabled parallel computing," IEEE Trans. Compon., Packag., Manuf. Technol., vol. 4, no. 10, pp. 1684-1695, Oct. 2014.

[9] $\mathrm{P} . \mathrm{Li}$ and L. J. Jiang, "Integration of arbitrary lumped multiport circuit networks into discontinuous Galerkin time-domain analysis," IEEE Trans. Microw. Theory and Techn., vol. 61, no. 7, pp. 2525-2534, Jul. 2013.

[10] P. Li, Y. Shi, L. J. Jiang, and H. Bagci, "A hybrid time-domain discontinuous galerkin-boundary integral method for electromagnetic scattering analysis," IEEE Trans. Antennas Propag., vol. 62, no. 5, pp. 2841-2846, May 2014.

[11] Q. Sun, Q. Zhan, Q. Ren, and Q. H. Liu, "Wave equation-based implicit subdomain DGTD method for modeling of electrically small problems," IEEE Trans. Microw. Theory Tech., vol. 65, no. 4, pp. 1111-1119, Apr. 2017.

[12] C. Fletcher, Computational Techniques for Fluid Dynamics. Berlin, Germany: Springer-Verlag, 1991.

[13] J. M. Jin, The Finite Element Method in Electromagnetics, 2nd ed. New York, NY, USA: Wiley, 2003.

[14] G. Meurant, "A review on the inverse of symmetric tridiagonal and block tridiagonal matrices," SIAM J. Matrix Anal. Appl., vol. 13, no. 3, pp. 707-728, Jul. 1992.

[15] P. Castillo, B. Cockburn, I. Perugia, D. Schotzau, "An a priori error analysis of the local discontinuous Galerkin method for elliptic problems," SIAM, vol. 38, no. 5, pp. 1676-1706, 2000.

[16] P. Li, Y. L. Dong, M. Tang, J. F. Mao, L. J. Jiang, and H. Bagci, "Transient thermal analysis of 3-D integrated circuits packages by the DGTD method," IEEE Trans. Compon., Packag., Manuf. Technol., vol. 7, no. 6, pp. 862-871, Jun. 2017.

[17] Y. L. Dong, M. Tang, P. Li, and J. F. Mao, "Transient electromagneticthermal simulation of dispersive media using DGTD method," IEEE Trans. Electromagn. Compat., vol. 61, no. 4, pp. 1305-1313, Aug. 2019.

[18] J. F. Chen, "An efficient discontinuous Galerkin finite element method with nested domain decomposition for simulations of microresistivity imaging,“ J. Applied Geophysics, vol. 114, pp. 116-122, 2015. 